\title{
Recruitment of Nanos to hunchback mRNA by Pumilio
}

\author{
Junichiro Sonoda and Robin P. Wharton ${ }^{1}$ \\ Howard Hughes Medical Institute (HHMI), Department of Genetics, Duke University Medical Center, \\ Durham, North Carolina 27710 USA
}

\begin{abstract}
Translational regulation of hunchback $(h b)$ mRNA is essential for posterior patterning of the Drosophila embryo. This regulation is mediated by sequences in the 3 '-untranslated region of $h b$ mRNA (the Nanos response elements or NREs), as well as two trans-acting factors-Nanos and Pumilio. Pumilio recognizes the NREs via a conserved binding motif. The mechanism of Nanos action has not been clear. In this report we use protein-protein and protein-RNA interaction assays in yeast and in vitro to show that Nanos forms a ternary complex with the RNA-binding domain of Pumilio and the NRE. Mutant forms of the NRE, Nos, and Pum that do not regulate $h b$ mRNA normally in embryos do not assemble normally into a ternary complex. In particular, recruitment of Nos is dependent on bases in the center of the NRE, on the carboxy-terminal Cys/His domain of Nos, and on residues in the eighth repeat of the Pum RNA-binding domain. These residues differ in a closely related human protein that also binds to the NRE but cannot recruit Drosophila Nos. Taken together, these findings suggest models for how Nos and Pum collaboratively target $h b$ mRNA. More generally, they suggest that Pum-like proteins from other species may also act by recruiting cofactors to regulate translation.
\end{abstract}

[Key Words: Drosophila; nanos; pumilio; RNA-binding protein; translational control]

Received August 13, 1999; revised version accepted September 3, 1999.

Post-transcriptional gene regulation is of particular importance in the early development of many organisms (Gray and Wickens 1998). During the rapid early nuclear cleavage cycles in Xenopus or Drosophila embryos, for example, the genome is transcriptionally inert; nevertheless, asymmetric patterns of gene expression arise as a result of the differential localization, stabilization, and translation of maternally synthesized mRNA. Translational regulation also plays a key role in germ-line sex determination in Caenorhabditis elegans (Goodwin et al. 1993; Zhang et al. 1997; Jan et al. 1999). In most of the cases studied in detail, signals in the $3^{\prime}$-untranslated region (UTR) of the appropriate mRNA mediate regulation. Recently, many of the trans-acting factors that bind to these signals have been identified, and a key issue is to understand how their binding effects regulation.

One such factor is the Drosophila pumilio protein (Pum). Pum binds to a pair of 32-nucleotide sequences (named Nanos response elements, NREs) in the $3^{\prime}$-UTR of maternal hunchback $(\bar{h} b)$ mRNA to repress its translation in the posterior of the embryo (Wharton and Struhl 1991; Murata and Wharton 1995). This translational repression is essential for normal abdominal segmentation (Wharton and Struhl 1991; Barker et al. 1992;

${ }^{1}$ Corresponding author.

E-MAIL rwharton@duke.edu; FAX (919) 681-8984.
Murata and Wharton 1995). The RNA-binding domain of Pum (Zamore et al. 1997; Wharton et al. 1998) is structurally similar to that of another translational regulator, FBF (fem-3 mRNA-binding factor) found in C. elegans (Zhang et al. 1997). The minimal RNA-binding domain of each protein consists of eight imperfect repeats plus flanking residues. These structural similarities define a

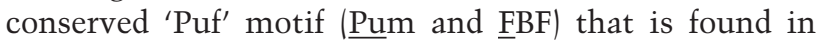
proteins from diverse organisms from yeast to humans (Zamore et al. 1997; Zhang et al. 1997). However, the RNA partner of no other Puf domain protein has been identified, nor is it clear whether other Puf proteins regulate translation or some other aspect of RNA metabolism.

Repression of $h b$ mRNA depends not only on Pum, but on the activity of the nanos protein (Nos). Nos is required for normal regulation of maternal $h b$ mRNA (Tautz 1988; Hülskamp et al. 1989; Irish et al. 1989; Struhl 1989). Whereas Pum is distributed uniformly throughout the embryo prior to fertilization (Macdonald 1992), Nos is selectively generated at the posterior pole during the initial stages of embryogenesis (Dahanukar and Wharton 1996; Smibert et al. 1996; Bergsten and Gavis 1999; Dahanukar et al. 1999). Thus, the distribution of Nos limits $h b$ translational regulation to the posterior of the embryo, thereby directing normal abdominal segmentation. The carboxy-terminal portion of Nos contains a divergent zinc-finger domain that has been 
reported to mediate nonspecific binding to RNA /Curtis et al. 1997). However, this activity is not sufficient to explain NRE-dependent regulation of $h b$ mRNA. No other biochemical functions have been assigned to Nos, and its role in the repression of $h b$ translation therefore has not been clear.

In this report, we show that Nos forms a specific ternary complex with Pum and the NRE. Mutations in Nos, Pum, or the NRE that specifically affect formation of this complex prevent normal regulation of $h b$ mRNA in the embryo. Thus, assembly of the ternary complex appears to be an essential step in translational control in vivo.

\section{Results}

\section{A ternary complex in yeast}

Previously we have shown that expression of the conserved RNA-binding domain of Pum (i.e., its Puf domain) is sufficient to rescue abdominal segmentation defects in otherwise pum $^{-}$embryos (Wharton et al. 1998). Thus, if Pum and Nos interact either directly or indirectly to regulate $h b$ mRNA, this interaction must be mediated by the Puf domain. However, we were unable to detect such an interaction using a variety of methods (including the yeast two-hybrid assay, coimmunoprecipitation, and affinity chromatography).

One explanation for this failure is that the Nos-Pum interaction is stabilized in the presence of the RNAbinding site (the NRE). To test this idea, we modified the three-hybrid RNA-binding assay (SenGupta et al. 1996) to assay ternary complex formation in yeast. A reporter strain in which HIS3 expression is driven by GAL4-binding sites was transformed with three plasmids encoding respectively: the Pum RNA-binding domain fused to the GAL4 DNA-binding domain (Pum-DBD), Nos fused to the GAL4 transcriptional activation domain (Nos-AD), and a chimeric nuclear RNA bearing NREs as well as binding sites for the bacteriophage MS2 coat protein (CP) (NRE/MS2) (Fig. 1A,B). Such triply transformed yeast grow on appropriate selective media lacking histidine, suggesting that Pum and the NRE collaboratively recruit Nos into a ternary complex. Triple transformants in which any one of the plasmids encoding Drosophila-derived components (Pum, Nos, or NRE) is substituted by empty vector do not grow on such media (Fig. 1B), consistent with the idea that only the ternary complex is sufficiently stable to detect with this assay. The yeast strain also contains $A D E 2$ and $l a c Z$ reporters under the control of GAL4-binding sites. These reporters respond in essentially the same manner as the HIS 3 reporter in all of the experiments described in this report (data not shown); for simplicity, we refer only to the latter results throughout.

In vitro, the carboxy-terminal domain of Nos has been reported to bind nonspecifically but with high affinity to RNA (Curtis et al. 1997). Thus, one interpretation of the results described above is that nonspecific binding of the Nos-AD fusion to Pum-bound RNA activates transcrip-
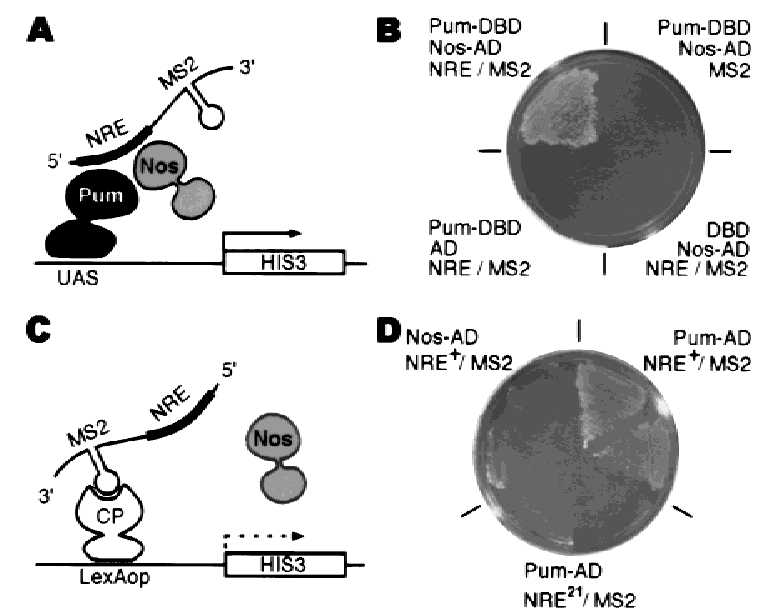

Figure 1. A Pum/NRE/Nos ternary complex in yeast. Experimental plans $(A, C)$ and results $(B, D)$. In $B$, yeast strains were transformed with plasmids that direct the synthesis of either hybrid molecules (i.e., Pum fused to GAL4-DBD) or empty vectors (i.e., DBD). Formation of the ternary complex $(A)$ activates transcription of HIS3, allowing growth in the absence of exogenous His $(B)$. In $C$ and $D$, a Nos-AD fusion does not bind to the NRE in the absence of Pum, and HIS3 transcription is not stimulated. As a control, the Pum-AD fusion binds to $\mathrm{NRE}^{+}$but not $\mathrm{NRE}^{21}$ in this assay (Fig. 2).

tion of HIS3. According to such a model, the Nos-AD fusion should activate HIS3 transcription if the NREbearing RNA is tethered to the promoter by a heterologous protein. However, as shown in Figure 1, C and D, Nos does not bind to the NRE when it is tethered by a fusion of the bacteriophage MS2 CP to a DBD. In contrast, Pum does bind to the wild-type NRE, but not to a mutant site, under such circumstances. Thus, Nos does not appear to form a stable binary complex with either the NRE or with Pum, suggesting that contacts to both molecules are required to form the ternary complex.

\section{Ternary complex formation depends on bases in the center of the NRE and amino acids in the carboxy-terminal domain of Nos}

Mutational analysis of NRE function in vivo and Pum binding in vitro has defined two classes of mutant sites (Wharton et al. 1998). One class is nonfunctional in vivo and binds Pum weakly or not at all in vitro (Fig. 2A). The second class is defective in mediating translational regulation in vivo, but binds Pum normally (Fig. 2A). One model consistent with these observations is that bases altered in this second class of mutants (at positions 1720 of the NRE, Fig. 2A) are required for recruitment of Nos.

To test this idea, the ability of various mutant NREs to support ternary complex formation was assayed in yeast, as described above. Ternary complex formation was observed only in yeast expressing the wild-type NRE; mutations in positions 17-20 of the NRE abolish expression of the HIS3 reporter, as do mutations in positions 21-22, which eliminate Pum binding in vitro (Fig. 2B). To test 
A

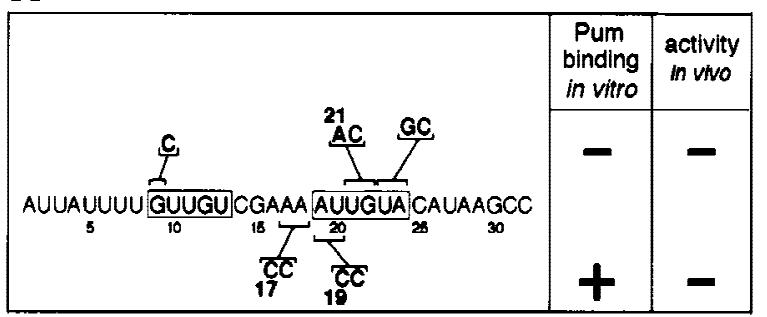

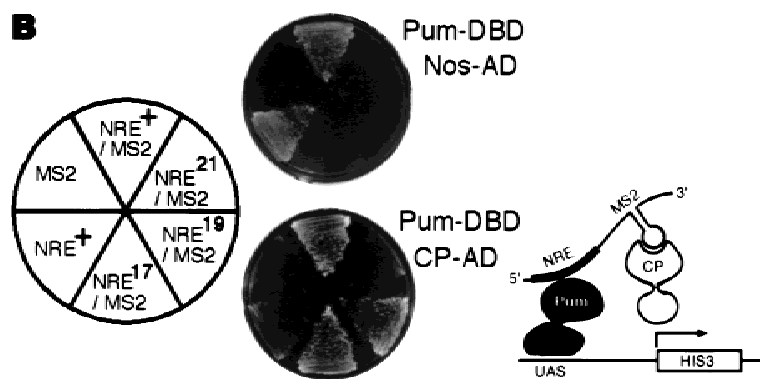
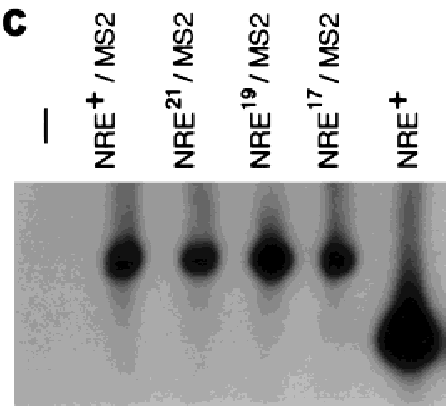

Figure 2. Bases in the center of the NRE are required to recruit Nos. $(A)$ Sequence of $\mathrm{NRE}^{+}$and mutants that reduce its activity in embryos (Murata and Wharton 1995; Wharton et al. 1998). Bases conserved among NREs in $b c d$ and $h b$ genes from various Drosophila species are boxed. Mutations above reduce binding of Pum and those below prevent incorporation of Nos into a ternary complex. Mutant NREs used in these studies, each bearing a dinucleotide substitution, are named according to their position. $(B)$ In vivo yeast assays of Pum/NRE/Nos ternary complex formation. Yeast expressing the Nos, Pum, or CP derivatives indicated were also transformed with plasmids that direct the synthesis of RNAs that contain either the MS2 hairpin (the CP-binding site), or the NRE, or both, as indicated in the drawing at left. The top plate monitors incorporation of Nos into a ternary complex, as shown schematically in Fig. 1A; the bottom plate monitors binding of Pum, as shown schematically in the drawing at right. $(C)$ Northern blot analysis to assay the level of RNA accumulation in yeast. Samples of RNA from yeast transformed either with empty vector (-) or plasmids that direct synthesis of each of the indicated chimeric RNAs were probed with NRE sequences. Each lane contains approximately the same amount of low-molecular-weight RNA as determined by staining with ethidium bromide. Note that the $\mathrm{NRE}^{+}$RNA (i.e., lacking the MS2 hairpin) accumulates to a higher level than do the NRE/MS2 chimeric RNAs. Compared with yeast expressing the $\mathrm{NRE}^{+} / \mathrm{MS} 2$ chimera (also cotransformed with Pum-DBD and Nos-AD plasmids), yeast expressing the $\mathrm{NRE}^{+}$RNA grow on His ${ }^{-}$media in the presence of higher levels of the HIS3 competitor 3-aminotriazole and express higher levels of LacZ (not shown). These observations suggest that the concentration of RNA substrate is limiting in the yeast ternary complex assay.

whether Pum can bind to these mutant NREs in yeast, a control experiment was performed in which a plasmid encoding $\mathrm{CP}-\mathrm{AD}$ was substituted for the Nos-AD plasmid (Fig. 2B). The results of this in vivo RNA-binding assay are consistent with in vitro-binding experiments: Pum binds to the mutant NREs bearing substitutions at positions 17-20 and not to the mutant bearing substitutions at positions 21-22. Northern blot analysis reveals that each NRE/MS2 chimeric RNA accumulates to a similar level (Fig. 2C).

To determine which portion of Nos mediates recruitment into the ternary complex, various deletion derivatives were tested as described above. Nos derivatives bearing the carboxy-terminal 97 amino acids of Nos are recruited into ternary complexes with the wild-type NRE but not a mutant NRE (Fig. 3A,B). In contrast, derivatives lacking some or all of the carboxy-terminal domain $(\Delta C, \Delta N 3)$ or one bearing the seven-amino-acid deletion in the carboxy-terminal tail encoded by $\operatorname{nos}^{L 7}$ (L7) do not. This mutant protein accumulates to normal levels in embryos (Wang et al. 1994) but is almost completely defective in regulating $h b$ translation (Wharton and Struhl 1991). Western blot analysis reveals that each Nos derivative is expressed at approximately the same level in yeast (Fig. 3C). Thus, the carboxy-terminal region of Nos mediates ternary complex formation.

\section{A region within the Pum RNA-binding domain} that is essential for ternary complex formation

Among the proteins that share structural similarity with
Drosophila Pum, one human homolog shows striking conservation, with $>80 \%$ identity throughout the RNAbinding domain (Zamore et al. 1997; Zhang et al. 1997). Moreover, this protein has been shown to bind with high affinity and specificity to the NREs in $h b$ mRNA (Zamore et al. 1997). Therefore, we asked whether the human Pum protein also shares the capacity to recruit Nos into a ternary complex.

Using the yeast assays described above (Figs. 1 and 2), we find that human Pum binds to the NRE in vivo but does not recruit Drosophila Nos into a ternary complex (Fig. 4). Because the human and fly Pum proteins are so similar, we imagined that chimeras would still bind to the NRE; if so, then we could determine whether a discrete portion of the fly protein is responsible for recruitment of Nos. As summarized in Figure 4, analysis of various chimeras reveals that amino acids within the eighth-repeat motif of the RNA-binding domain are responsible for the difference in activity of the two Pum proteins. In particular, the human protein bears an insertion relative to the fly protein of three amino acids (GPH) in this region. A human derivative lacking the GPH residues recruits fly Nos into a ternary complex, whereas a fly derivative bearing an insertion of the GPH residues does not. All of the chimeras tested bind specifically to the wild type but not to the $\mathrm{NRE}^{21}$ mutant (Fig. 4). Thus, residues in the eighth-repeat motif of the RNA-binding domain impart specificity to the Pum-Nos interaction during formation of the ternary complex.

If the ternary complex is biologically relevant, then mutations that disrupt its formation should prevent nor- 
Pumilio recruits Nanos

A

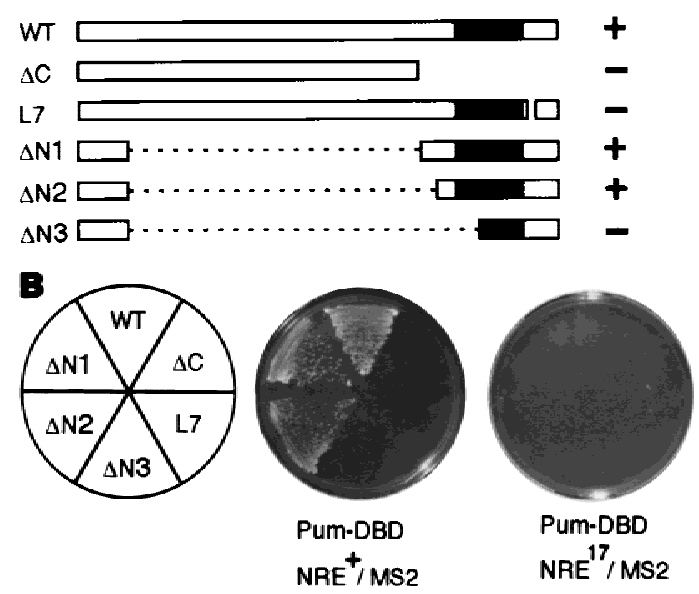

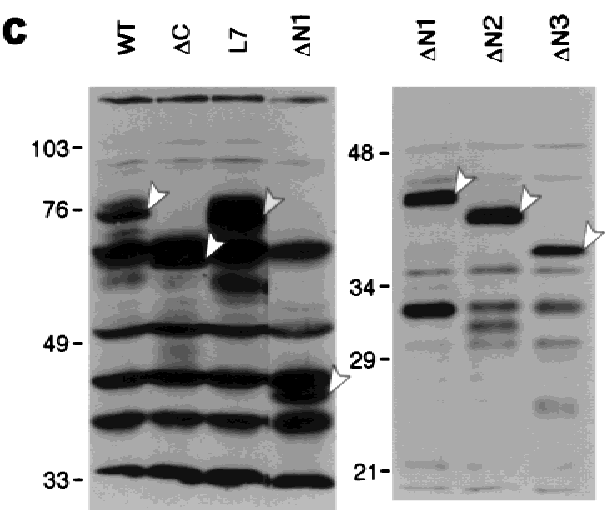

Figure 3. The carboxy-terminal portion of Nos mediates recruitment into the ternary complex. (A) Drawings (to scale) of Nos derivatives tested for ternary complex formation in yeast. The conserved Cys/His domain is filled in. Results, shown in $B$, are summarized at right. Note that deletion of residues at the amino terminus of Nos (i.e., amino acids 1-42 at the junction with the $\mathrm{AD}$ ) abolishes activity for unknown reasons, even though these are dispensable for nos function in vivo, as determined using a suitably modified transgene (data not shown). Note also that residues 1-42 are not present in the His6-Nos protein used to assay ternary complex formation in vitro (Fig. 6). (C) Western blot of yeast extracts prepared from transformants expressing each of the indicated Nos-AD derivatives. The blots were probed with a monoclonal anti-HA antibody that recognizes a vectorencoded epitope tag. Each derivative (arrowhead) accumulates to a level equal to or greater than the level of the wild-type (WT) fusion, with the exception of the $\Delta \mathrm{N} 3$ derivative, which accumulates to a slightly lower level. For the blot at right, the antibody was preadsorbed and the background is lower as a result.

mal regulation of $h b$ mRNA in embryos. As described above, this correlation holds for mutations in Nos (L7, Fig. 3) and the NRE (bases 17-20, Fig. 2). We wished to determine whether the correlation also holds for mutations in Pum that specifically affect recruitment of Nos without affecting RNA-binding activity. In previous work (Wharton et al. 1998), we identified a derivative of Pum, here named Pum ${ }^{\mathrm{Mlu}}$, which bears a four-aminoacid insertion at essentially the same position in the RNA-binding domain where insertion of GPH (derived from human Pum) blocks Nos recruitment (Fig. 4). Further experiments were performed using the Pum ${ }^{\text {Mlu }} \mathrm{mu}$ tant, as described below.

When tested in the yeast assays described above, the Pum ${ }^{\text {Mlu }}$ mutant binds normally to the NRE, but does not recruit Nos into a ternary complex (Fig. 5A). This defect appears to be specific, as another mutant, Pum ${ }^{60}$ (which bears a single amino acid substitution in the seventh repeat of the RNA-binding domain) (Wharton et al. 1998), binds to the NRE and recruits Nos normally (Fig. 5A). Western blot analysis reveals that each Pum derivative accumulates to a similar level in yeast (Fig. 5B).

To test its activity in vivo, transgenic flies that express the Pum ${ }^{\text {Mlu }}$ mutant RNA-binding domain (plus carboxyterminal residues) were prepared. The otherwise identical Pum ${ }^{+}$and Pum ${ }^{\mathrm{Mlu}}$ protein fragments accumulate to the same level in embryos (Fig. 5C). Moreover, each of these proteins binds specifically to wild-type but not mutant NREs in vitro, as determined in UV cross-linking experiments using extracts prepared from transgenic

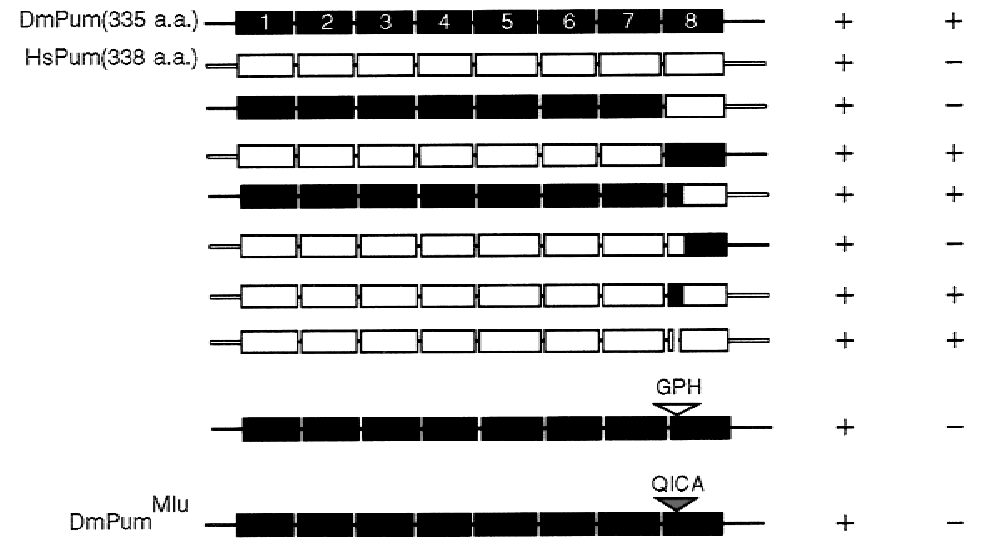

Figure 4. Human Pum binds to the NRE but does not recruit Nos. Shown are drawings (to scale) of the RNA-binding domains of Drosophila Pum (DmPum), human Pum (HsPum), and various chimeric proteins. The results of yeast in vivo interaction assays are summarized at right, with specific binding to $\mathrm{NRE}^{+}$ (and not $\mathrm{NRE}^{21}$ ) assayed as in Fig. 2B and recruitment of Nos into a ternary complex assayed as in Fig. 1A. Each of the eight repeats that comprise the core of the RNA-binding domain is indicated by a box, which is labeled only on the first line for clarity. 
Figure 5. A Pum mutant that cannot recruit Nos does not regulate $h b$ mRNA in vivo. $(A)$ In vivo yeast assays of NRE binding (left) and Nos recruitment (right) by wild-type Pum and two mutants. Experimental designs for these experiments are shown schematically in Figs. 2B and $1 \mathrm{~A}$, respectively. Yeast express the chimeric molecules shown below as well as the Pum derivatives shown at left. $(B)$ Western blot of samples prepared from yeast transformed with empty vector (-) or plasmids encoding each of the indicated Pum/DBD fusions probed with antiPum antibodies. Although there is less $\mathrm{Pum}^{680}$ than $\mathrm{Pum}^{+}$in vivo, evidently the level of this mutant protein is saturating, as both transformants grow at a similar rate on $\mathrm{His}^{-}$plates containing various 3-AT concentrations, and both stimulate the lacZ reporter to a similar extent (data not shown). Note that overproduction of $\mathrm{Pum}^{+}$also results in the accumulation of lower-molecular-weight (i.e., $<49 \mathrm{kD}$ ) apparent breakdown products, which are visible for Pum ${ }^{\text {Mlu }}$ in lane 3 (not shown). (C) Western blot of control wild-type (WT) or transgenic embryos expressing the RNA-binding domain fragments described in the text and indicated above. The blot was probed with anti-Pum antibodies, which recognize both the endogenous full-length protein (upper arrow) as well as the protein encoded by the transgene (lower arrow). (D) UV crosslinking of extracts prepared from wild-type control or transgenic embryos expressing either Pum ${ }^{+}$or Pum ${ }^{\text {Mlu }}$ RNA-binding domain derivatives, as indicated. In each pair of lanes, an aliquot of each extract was incubated with labeled $\mathrm{NRE}^{+}$(left) or $\mathrm{NRE}^{21}$ (right) RNA. The upper arrow indicates the endogenous full-length Pum protein and the lower arrow indicates the transgene-encoded RNA-binding domain. $(E)$ Each line shows the distribution of $\mathrm{Hb}$ in the early embryo in a midline, lateral view (left) and a dark-field photograph of the cuticle secreted by the mature embryo in the vitelline membrane in a ventral surface view (right). Embryos are derived from pum ${ }^{-}$females (top row) or pum ${ }^{-}$females that express the $\mathrm{Pum}^{+}$or Pum ${ }^{\mathrm{Mlu}}$ RNA-binding domain fragments from transgenes (middle and bottom rows, as indicated). Embryos are oriented anterior at left. Note that only in the posterior half of the embryo is $\mathrm{Hb}$ accumulation derived solely from translation of maternal mRNA; in the anterior, Bicoid-dependent zygotic transcription of $\mathrm{hb}$ also contributes. Note also that in the $\mathrm{pum}^{E T 3} / \mathrm{pum}^{\mathrm{Msc}}$ background weak residual pum activity is evident at earlier stages of embryogenesis. In the right column, abdominal segmentation (prominent bands of thick hairs that appear as white dots) is apparent only in the middle panel.

embryos (Fig. 5D). To determine whether these proteins can regulate translation of $h b$ mRNA, transgenes were introduced into otherwise pum females, and the distribution of $h b$ protein was examined in embryos derived from these females. As shown in Figure 5E, in the pum control embryos, maternal $\mathrm{Hb}$ mRNA is not repressed, $h b$ accumulates throughout the posterior half of the embryo, and no abdominal segments develop in consequence. In contrast, expression of the $\mathrm{Pum}^{+}$RNA-binding domain substantially represses the accumulation of $\mathrm{Hb}$ in the posterior and abdominal segmentation is largely rescued, as reported previously (Wharton et al. 1998). The Pum ${ }^{\text {Mlu }}$ RNA-binding domain does not block accumulation of $\mathrm{Hb}$ in the posterior of the embryo, and no abdominal segments develop as a result.

In summary, the Pum ${ }^{\text {Mlu }}$ mutant binds RNA normally but cannot recruit Nos into a ternary complex with the NRE in yeast and cannot regulate $h b$ translation normally in the embryo. Taken together, these observations support the idea that formation of the ternary complex is essential for the Nos- and Pum-dependent regulation of $h b$ in vivo.

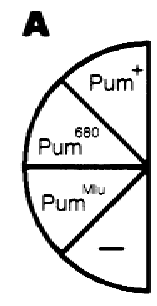

A

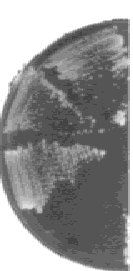

NRE / MS2 CP-AD

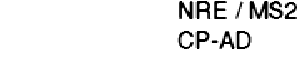

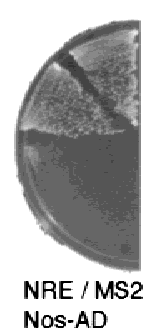

Nos-AD
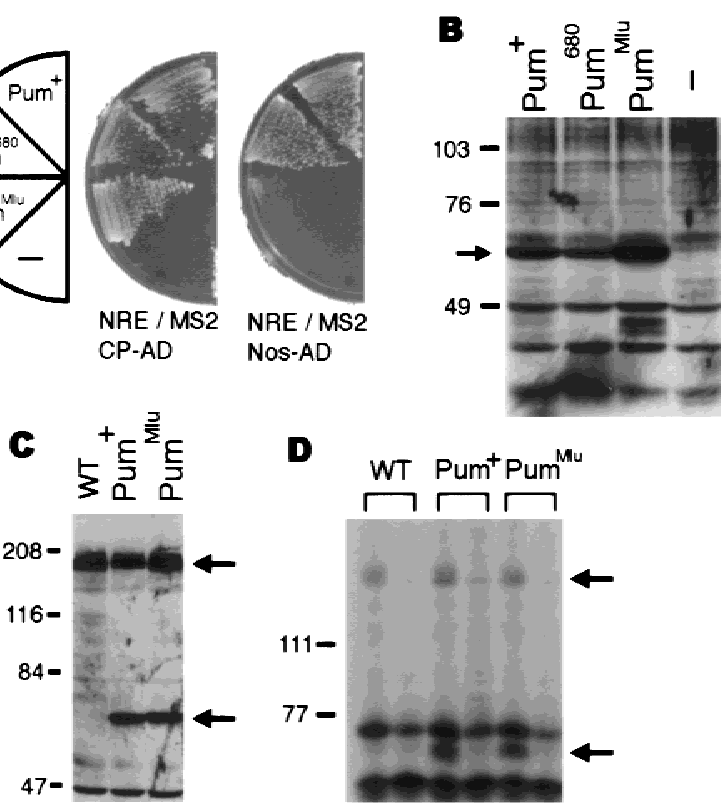

$\mathbf{E}$
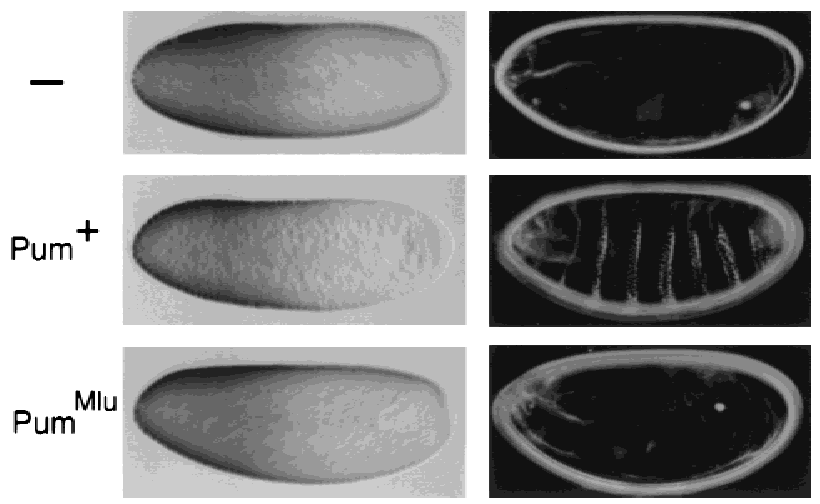

Ternary complex formation in vitro

We next wished to extend the in vivo interaction experiments described above to rule out the possibility that yeast proteins or RNAs might mediate formation of the Nos/Pum/NRE ternary complex. Accordingly, NREbearing RNA was synthesized by in vitro transcription, a glutathione $S$-transferase fusion to the RNA-binding domain of Pum (GST-Pum) (Wharton et al. 1998) was prepared from bacteria, and a hexa-His-tagged derivative of the carboxy-terminal region of Nos (His6-Nos) was also prepared from bacteria. These purified components, or mutant derivatives, were mixed together, and reaction mixtures were incubated with glutathione-agarose beads. Ternary complex formation was assayed by retention of Nos, which does not bind to glutathione-agarose on its own (data not shown; see Fig. 6).

As shown in Figure $6 \mathrm{~A}, \sim 5 \%-10 \%$ of the full-length input Nos is retained under the conditions of these experiments when it is incubated in the presence of wild type GST-Pum and the wild-type NRE. During purification, the His6-Nos protein suffers proteolytic degrada- 
A
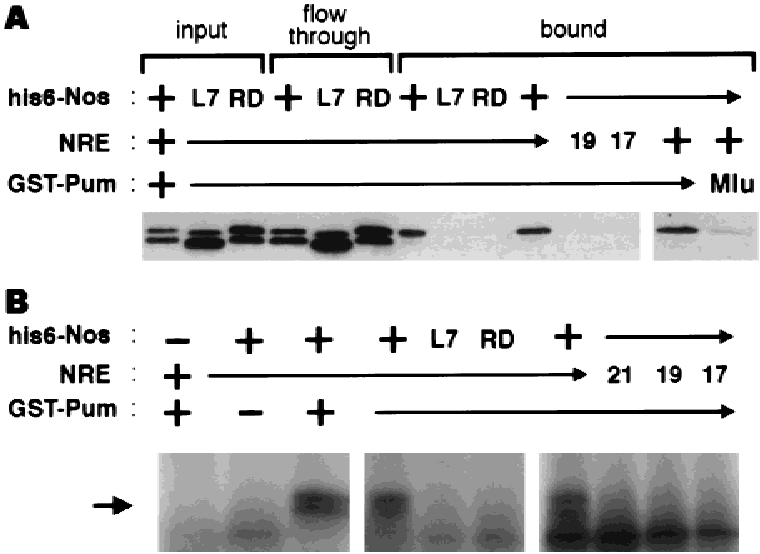

Figure 6. Ternary complex formation in vitro. (A) Purified His6-Nos is visualized by Western blot using an antibody that recognizes the amino-terminal Xpress tag. This protein is degraded during purification from bacteria, yielding a doublet. In each reaction, either the wild-type molecule $(+)$ or the indicated mutant derivative was incubated in a binding reaction, and ternary complexes were captured on glutathione-agarose beads, as described in Materials and Methods. Note that only the longer His6-Nos derivative is incorporated into ternary complexes. Lanes 1-3 contain $10 \%$ of the input, lanes $4-6$ contain $10 \%-$ $15 \%$ of the flowthrough, and lanes $7-14$ contain $100 \%$ of the bound fraction. $(B)$ The results of UV cross-linking experiments in which covalent RNA-protein adducts are detected by autoradiography following preincubation of the indicated mixture of molecules (above each lane) and UV irradiation. The RNA crosslinks to both Pum and Nos; because the latter reaction is very inefficient, the signal from the Pum-RNA adduct was removed and only a portion of the autoradiogram is shown. The NosRNA adduct (arrow) is a doublet, presumably because of heterogeneity in the length of the RNA. Note that an impurity in the His6-Nos preparation cross-links nonspecifically to both wildtype and mutant RNAs (visible at bottom).

tion at the carboxyl terminus; as a consequence, the preparation consists of a roughly equimolar mixture of full-length and carboxy-terminally truncated protein. As only the full-length protein is retained in ternary complexes, residues near the carboxyl terminus appear to be required for recruitment by Pum and the NRE.

Next, the ability of various mutant forms of Nos, Pum, and the NRE to form ternary complex was tested. As shown in Figure 6A, retention of Nos is substantially reduced if any of the reaction components bears substitutions that reduce or eliminate $h b$ regulation in the embryo. In particular retention of His6-Nos is reduced: (1) at least 10-fold using NRE mutants bearing substitutions at positions 17-20 (Fig. 2A); (2) at least 10-fold if it bears the L7 mutation (Fig. 3) or the RD mutation that results in substitution of Tyr for one of the conserved Cys residues (Curtis et al. 1997); and (3) at least threefold by the Mlu mutation in Pum (Figs. 4 and 5). The Nos ${ }^{\mathrm{L} 7}$ mutant protein lacks seven amino acids near the carboxyl terminus (Curtis et al. 1997), supporting the idea that residues in this region are essential for recruitment. Thus, formation of the ternary complex depends on the integrity of each Drosophila-derived component and not on extraneous proteins or RNAs in yeast.
The carboxy-terminal portion of Nos that is required for ternary complex formation (Fig. 3) has been reported to bind nonspecifically to RNA in the absence of cofactors (Curtis et al. 1997). To determine whether Nos contacts the RNA on recruitment into the ternary complex, ${ }^{32}$ P-labeled NRE-bearing RNA was incubated with GSTPum and His6-Nos to form ternary complexes, and the mixture was UV-irradiated to cross-link proteins to RNA. Following RNase digestion, proteins were separated by SDS-PAGE, and covalent RNA-protein adducts detected by autoradiography. As shown in Figure 6B, Nos-RNA adducts are detectable when wild type proteins and RNA are incubated together, but not when Pum is omitted from the reaction or when mutant forms of Nos or the NRE are used (Fig. 6B). Thus, Nos appears to contact RNA directly within the ternary complex.

\section{Discussion}

Using both yeast in vivo interaction assays and purified components in vitro, we find that Nos interacts specifically with Pum and the NRE to form a ternary complex. Binary complexes between Nos and the NRE or between Nos and Pum are not detectable under otherwise identical conditions in either assay. Mutations in Nos, Pum, and the NRE that prevent normal regulation of $h b$ in embryos reduce or eliminate formation of the ternary complex. Therefore, recruitment of Nos jointly by Pum and the NRE appears to explain how $h b$ mRNA is specifically targeted in vivo. These results provide a basis for understanding the mechanism by which Nos and Pum collaborate to regulate translation.

Figure 7 shows two working models of how the ternary complex might assemble. Pum and maternal $h b$ mRNA (bearing the NRE) are present in the embryo at fertilization. Pum binds specifically to the NRE in the absence of cofactors (including Nos) (Murata and Wharton 1995; Zamore et al. 1997; Wharton et al. 1998), and measurements of its affinity and concentration suggest that Pum likely saturates the NREs in the embryo (Zamore et al. 1999). Analysis of mutant NREs in vivo and in vitro suggests that Pum primarily recognizes bases at each end of the site and not those in the center (Wharton et al. 1998). This idea is further supported by the observation that purified Pum binds weakly to molecules containing each half of the NRE. Following fertilization, newly synthesized Nos is recruited by the Pum/NRE complex. Figure 7 indicates two models for the resulting ternary complex.

In one model, Nos simultaneously makes specific contacts with Pum and nucleotides 17-20 of the NRE. On their own, neither the Nos-Pum nor the Nos-NRE contacts are strong enough to recruit Nos to $h b$ mRNA (at least in the presence of competitor proteins and RNAs), because binary complexes with Nos are not detectable. In another model, unbound Pum cannot interact with Nos, but binding to the NRE induces a conformational change in Pum, which subsequently recruits Nos via protein-protein contacts. In this model, nucleotides 1720 of the NRE interact with Pum to induce the confor- 


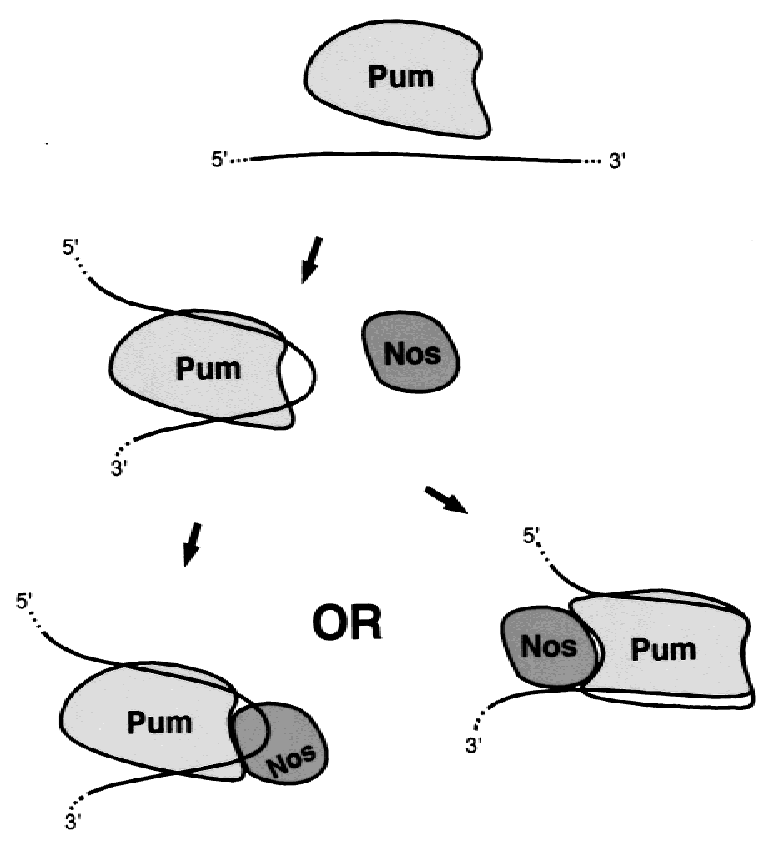

Figure 7. Models of ternary complex formation in the embryo. At left, Nos is recruited by a combination of weak but specific protein-protein and protein-RNA contacts. At right, Nos is recruited primarily by specific contacts to a surface of Pum that is competent only on binding to the NRE, although nonspecific contacts between Nos and the RNA may also stabilize the ternary complex.

mational change without affecting its affinity for the RNA, and nonspecific interactions between Nos and other portions of the RNA help stabilize the complex. Either model is consistent with the nonspecific RNAbinding activity reported for the carboxy-terminal portion of Nos in vitro (Curtis et al. 1997) and the RNA-Nos cross-link reported in Figure 6B. Further structural and biochemical experiments will be required to distinguish between these (or alternative) models.

The mechanism by which the ternary complex blocks translation is not yet clear. Earlier studies showed that mRNAs subject to Nos- and Pum-dependent repression are deadenylated in vivo (Wharton and Struhl 1991; Wreden et al. 1997). In addition, Nos and Pum have been shown to regulate internal ribosome entry site (IRES)dependent translation in imaginal disc cells, suggesting that their regulatory target lies downstream of cap recognition and scanning (Wharton et al. 1998). We assume that some surface of the ternary complex, formed jointly by Nos and Pum, targets a component of the polyadenylation or translation machinery. This surface appears to be altered in the Pum ${ }^{680}$ mutant protein, which binds the NRE normally but is defective in regulating $h b$ translation in the embryo (Wharton et al. 1998). The Pum ${ }^{680}$ mutant recruits Nos into a ternary complex normally (Fig. 5) and thus apparently is defective in a subsequent step of the repression reaction. The RNA-binding domain of Pum therefore appears to have at least three different functions in regulating $h b$-recognizing the
NRE, recruiting Nos, and acting as a corepressor (with Nos) to block translation.

In the experiments reported here, we focus on discrete regions of both Nos (the carboxy-terminal 97 amino acids) and Pum (the minimal RNA-binding domain), which play an essential role in formation of the ternary complex. However, other regions of Nos are required for its function in repressing translation in the embryo (Curtis et al. 1997). In addition, residues elsewhere in Pum play an unknown role in augmenting the intrinsic translational repression activity of the RNA-binding domain (Wharton et al. 1998). Thus, the ternary complex formed by the 157-kD, full-length Pum protein may be stabilized by auxillary protein-protein or protein-RNA interactions in addition to those that mediate recruitment of the carboxy-terminal domain of Nos by the RNA-binding or Puf domain of Pum.

The results described above suggest that Puf domain proteins generally may act by recruiting cofactors to specific RNA binding sites. Cofactor specificity may be mediated, at least in part, by the eighth repeat of the Puf domain (Fig. 4). Although Puf domain proteins have been described in organisms from yeast to humans, for only one protein other than Drosophila Pum, C. elegans FBF, is the relevant RNA regulatory target known. FBF regulates the sperm/oocyte switch in the hermaphrodite germ line by governing the translation of fem-3 mRNA (Zhang et al. 1997). Kimble, Wickens, and colleagues have found recently that the FBF RNA-binding domain interacts with one of the C. elegans Nos homologs (Kraemer et al. 1999). Further experiments will be required to determine whether the Pum/fly Nos complex and the $\mathrm{FBF} /$ worm Nos complex function in a similar manner.

\section{Materials and methods}

\section{Fly strains and reagents}

Transgenes were constructed in PCasPeR derivatives and introduced into $w^{1118}$ flies by standard methods. The transgenes used in Figure 5 were pum $\left(\Delta 2^{\prime}\right)$ and an isogenic derivative encoding Pum $^{\text {Mlu }}$ (Wharton et al. 1998). At least three independent transgenic lines were crossed into pum $^{-}$backgrounds. Abdominal segmentation was assayed in both $\mathrm{pum}^{\mathrm{Msc}} / \mathrm{pum}^{\text {ET3 }}$ and $\mathrm{pum}^{\mathrm{Msc}} / \mathrm{pum}^{F C 8}$ transheterozygous backgrounds, whereas the distribution of $\mathrm{Hb}$ was examined only in the former. These alleles were a gift from Ruth Lehmann. DNA encoding human Pum was prepared by repairing a clone received from Phil Zamore that bears the sequence ACAGAGCAGCTGGTACAGATCAATAT in the open reading frame (see Zamore et al. 1997 for details). Antibodies that recognize $\mathrm{Hb}$ were a gift from Paul Macdonald, antibodies that recognize the HA tag encoded by pAct 2 were from Roche, and antibodies that recognize Pum were from animals injected with a GST fusion to residues 10931533 of Pum.

\section{Yeast interaction assays}

PJ69-4A (James et al. 1996) was used in all of the experiments except those described in Figure 1, C, and D, which use L40-coat (SenGupta et al. 1996). Transformants were restreaked onto $\mathrm{His}^{-}$medium containing 0-7 mM 3-aminotriazole or $\mathrm{Ade}^{-}$me- 
dium (not shown). A fragment encoding residues 1093-1426 of Pum was inserted into pGBT9 or pACT2 (Clontech) to generate the Pum-DBD and Pum-AD plasmids. An additional enhancer was inserted into the Pum ${ }^{\mathrm{Mlu}}$-DBD plasmid to boost expression of this derivative. Fragments encoding the entirety of CP (residues 1-129) and the entirety of Nos (residues 1-401) were prepared by PCR and inserted into pACT2. Two copies of a DNA fragment encoding each of various NREs were inserted into the SpeI site of pIII/MS2-1 (SenGupta et al. 1996) to generate the NRE/MS2 plasmids. Note that a potential RNA Pol III termination signal present in a nonessential portion of the NRE (UUUU) (Wharton et al. 1998) was replaced by UUAU. In Figure 3, residues deleted in the Nos derivatives are as follows: $\Delta \mathrm{C}(288-401), \mathrm{L} 7(376-382)$ (Curtis et al. 1997), $\Delta \mathrm{N} 1(43-287)$, $\Delta N 2(43-304), \Delta N 3(43-336)$. Plasmids encoding fusions to the GAL4 DBD of the fly/human chimeras of Figure 4 were constructed by standard PCR techniques. The 'parental' fly protein at the top of the figure contains the 335 amino acids that comprise the minimal RNA-binding domain /residues 1093-1427 of full-length Pum). The corresponding region of the parental human protein on the second line contains a three-amino-acid insertion and thus is 338 amino acids long. Because the human clone is a fragment, numbering is with respect to the corresponding residues of the RNA-binding domain of fly Pum. In the following list, residues derived from fly Pum are in bold, and insertions are listed in single-letter code. From the third line of Figure 4 , the Pum derivatives contain the following residues: 1-275, 276-338; 1-274, 275-335; 1-282, 286-338; 1-285, 283335; 1-274, 275-282, 286-338; 1-277, 281-338; 1-277, GPH, 278-335; 1-279, QICA, 280-335. For the Northern blot of Figure 2C, low-molecular-weight RNA was prepared from various yeast transformants, electrophoresed through a denaturing acrylamide gel, electroblotted, and probed either with RPR sequences (not shown) or a cocktail of $\mathrm{NRE}^{+}$and mutant NRE sequences.

\section{Ternary complex formation in vitro}

Plasmids encoding GST-Pum and NRE-bearing RNAs are described elsewhere (Murata and Wharton 1995; Wharton et al. 1998). The plasmid encoding His6-Nos was constructed by insertion of a fragment encoding residues 288-401 of Nos into pRSET (Invitrogen). RNA was prepared by in vitro transcription as described previously (Murata and Wharton 1995), and proteins were partially purified by affinity chromatography on glutathione-agarose (Sigma) and $\mathrm{Ni}^{2+}-\mathrm{NTA}$ agarose (Qiagen) according to the manufacturer's instructions. Western blot analysis reveals that the His6-Nos in bacteria prior to lysis and purification comigrates with the slower-moving band of Figure 6A. Because the His6 and Xpress tags are at the amino terminus of the protein, we infer that the faster-migrating band results from proteolysis near the carboxyl terminus. GST-Pum (3 $\mu \mathrm{M})$ and unlabeled RNA $(2 \mu \mathrm{M})$ were mixed in interaction buffer $(20$ mM HEPES, $5 \mathrm{~mm} \mathrm{MgCl}_{2}, 5 \mu \mathrm{ZnCl}_{2}, 0.5 \mathrm{~mm}$ DTT, $100 \mathrm{~mm}$ $\mathrm{NaCl}, 0.1 \%$ Tween 20 ) containing $0.1 \%$ BSA, $500 \mathrm{U} / \mu \mathrm{l}$ RNase inhibitor (Roche), $1 \times$ proteinase inhibitor mix (Murata and Wharton 1995; Wharton et al. 1998) and incubated at room temperature for $10 \mathrm{~min}$. His6-Nos (1 $\mathrm{\mu m}$ ) was added, and the reaction incubated for $5 \mathrm{~min}$ at room temperature and then an additional $30 \mathrm{~min}$ at $4^{\circ} \mathrm{C}$. Glutathione-agarose beads (Sigma) were added and the reaction was incubated for a further $30 \mathrm{~min}$ at $4^{\circ} \mathrm{C}$ with agitation. Beads were washed three times with interaction buffer, and bound proteins were eluted by boiling in SDS-gel loading buffer. His6-Nos protein was detected with monoclonal anti-Xpress antibodies (Invitrogen) that recognize an amino-terminal tag. The fraction of bound protein was estimated by com- paring signal intensity with a serial dilution of input protein. RNAs containing both the $5^{\prime}$ and $3^{\prime}$ NRE from $h b$ were used in these experiments (Fig. 6A, lanes 1-9 and 10-14, respectively); no significant difference in activity was detectable. The specific activities of the GST-Pum ${ }^{+}$and GST-Pum ${ }^{\text {Mlu }}$ preparations were indistinguishable in gel mobility shift experiments (not shown). UV cross-linking was carried out in the interaction buffer described above except that $\mathrm{KCl}$ was substituted for $\mathrm{NaCl}$ and poly $(\mathrm{U})$ and $\operatorname{poly}(\mathrm{C})$ competitors were added to a concentration of $0.1 \mathrm{mg} / \mathrm{ml}$.

\section{Acknowledgments}

We thank Marvin Wickens and Joe Heitman for reagents; Brian Kraemer, Judith Kimble, and Marv Wickens for discussions of their results prior to publication; Bryan Cullen and Dan Kiehert for critical comments on the manuscript; Tammy Lee, Michelle Patterson, and Sherry Franklin for technical assistance; Glenda Johnson for media and fly food preparation; Sandy Boyles for secretarial assistance; and members of the laboratory for suggestions. J.S was supported in part by a scholarship from the Ishizaka Foundation. R.P.W. is an Assistant Investigator of HHMI.

The publication costs of this article were defrayed in part by payment of page charges. This article must therefore be hereby marked 'advertisement' in accordance with 18 USC section 1734 solely to indicate this fact.

\section{References}

Barker, D.D., C. Wang, J. Moore, L.K. Dickinson, and R. Lehmann. 1992. Pumilio is essential for function but not for distribution of the Drosophila abdominal determinant Nanos. Genes \& Dev. 6: 2312-2326.

Bergsten, S.E. and E.R. Gavis. 1999. Role for mRNA localization in translational activation but not spatial restriction of nanos RNA. Development 126: 659-669.

Curtis, D., D.K. Treiber, F. Tao, P.D. Zamore, J.R. Williamson, and R. Lehmann. 1997. A CCHC metal-binding domain in Nanos is essential for translational regulation. EMBO $J$. 16: $834-843$.

Dahanukar, A. and R.P. Wharton. 1996. The Nanos gradient in Drosophila embryos is generated by translational regulation. Genes \& Dev. 10: 2610-2620.

Dahanukar, A., J.A. Walker, and R.P. Wharton. 1999. Smaug, a novel RNA-binding protein that operates a translational switch in Drosophila. Mol. Cell 4: 209-218.

Goodwin, E.B., P.G. Okkema, T.C. Evans, and J. Kimble. 1993. Translational regulation of tra- 2 by its $3^{\prime}$ untranslated region controls sexual identity in C. elegans. Cell 75: 329-339.

Gray, N.K. and M. Wickens. 1998. Control of translation initiation in animals. Annu. Rev. Cell Dev. Biol. 14: 399-458.

Hülskamp, M., C. Schröder, C. Pfeifle, H. Jäckle, and D. Tautz. 1989. Posterior segmentation of the Drosophila embryo in the absence of a maternal posterior organizer gene. Nature 338: 629-632.

Irish, V., R. Lehmann, and M. Akam. 1989. The Drosophila posterior-group gene nanos functions by repressing hunchback activity. Nature 338: 646-648.

James, P., J. Halladay, and E.A. Craig. 1996. Genomic libraries and a host strain designed for highly efficient two-hybrid selection in yeast. Genetics 144: 1425-1436.

Jan, E., C.K. Motzny, L.E. Graves, and E.B. Goodwin. 1999. The STAR protein, GLD-1, is a translational regulator of sexual 
identity in Caenorhabditis elegans. EMBO J. 18: 258-269.

Kraemer, B., S. Crittenden, M. Gallegos, G. Moulder, R. Barstead, J. Kimble, and M. Wickens. 1999. NANOS-3 and FBF proteins physically interact to control the sperm/oocyte switch in C. elegans. Curr. Biol. (in press).

Macdonald, P.M. 1992. The Drosophila pumilio gene: An unusually long transcription unit and an unusual protein. Development 114: 221-232.

Murata, Y. and R.P. Wharton. 1995. Binding of pumilio to maternal hunchback mRNA is required for posterior patterning in Drosophila embryos. Cell 80: 747-756.

SenGupta, D.J., B. Zhang, B. Kraemer, P. Pochart, S. Fields, and M. Wickens. 1996. A three-hybrid system to detect RNAprotein interactions in vivo. Proc. Natl. Acad. Sci. 93: 84968501.

Smibert, C.A., J.E. Wilson, K. Kerr, and P.M. Macdonald. 1996. smaug protein represses translation of unlocalized nanos mRNA in the Drosophila embryo. Genes \& Dev. 10: 26002609.

Struhl, G. 1989. Differing strategies for organizing anterior and posterior body pattern in Drosophila embryos. Nature 338: 741-744.

Tautz, D. 1988. Regulation of the Drosophila segmentation gene hunchback by two maternal morphogenetic centres. Nature 332: 281-284.

Wang, C., L.K. Dickinson, and R. Lehmann. 1994. Genetics of nanos localization in Drosophila. Dev. Dyn. 199: 103-115.

Wharton, R.P. and G. Struhl. 1991. RNA regulatory elements mediate control of Drosophila body pattern by the posterior morphogen nanos. Cell 67: 955-967.

Wharton, R.P., J. Sonoda, T. Lee, M. Patterson, and Y. Murata. 1998. The Pumilio RNA-binding domain is also a translational regulator. Mol. Cell 1: 863-872.

Wreden, C., A.C. Verrotti, J.A. Schisa, M.E. Lieberfarb, and S. Strickland. 1997. Nanos and pumilio establish embryonic polarity in Drosophila by promoting posterior deadenylation of hunchback mRNA. Development 124: 3015-3023.

Zamore, P.D., J.R. Williamson, and R. Lehmann. 1997. The Pumilio protein binds RNA through a conserved domain that defines a new class of RNA-binding proteins. RNA 3: 14211433.

Zamore, P.D., D.P. Bartel, R. Lehmann, and J.R. Williamson. 1999. The PUMILIO-RNA interaction: A single RNA-binding domain monomer recognizes a bipartite target sequence. Biochemistry 38: 596-604.

Zhang, B., M. Gallegos, A. Puoti, E. Durkin, S. Fields, J. Kimble, and M.P. Wickens. 1997. A conserved RNA-binding protein that regulates sexual fates in the C. elegans hermaphrodite germ line. Nature 390: 477-484. 


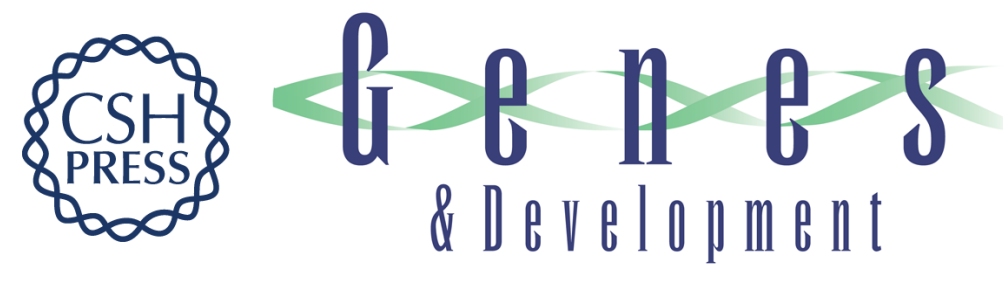

\title{
Recruitment of Nanos to hunchback mRNA by Pumilio
}

\author{
Junichiro Sonoda and Robin P. Wharton
}

Genes Dev. 1999, 13:

References This article cites 24 articles, 11 of which can be accessed free at: http://genesdev.cshlp.org/content/13/20/2704.full.html\#ref-list-1

\section{License}

Email Alerting

Receive free email alerts when new articles cite this article - sign up in the box at the top Service right corner of the article or click here.

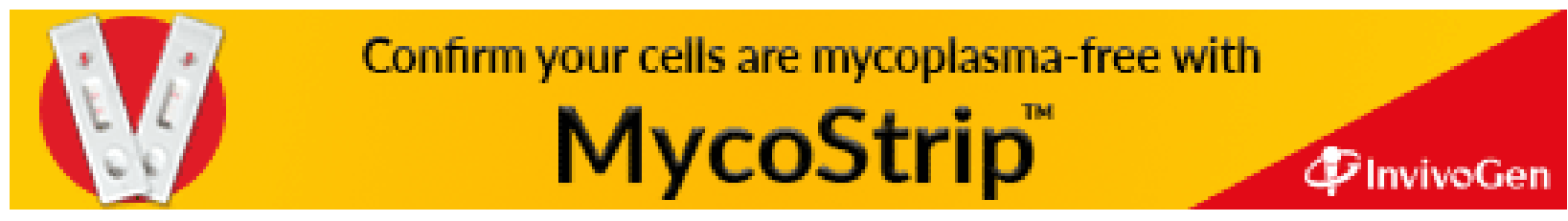

